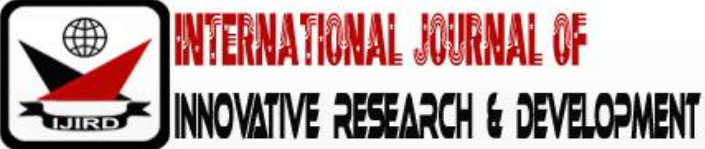

ISSN 2278 - 0211 (Online)

\section{Prevalence of Elevated and Hypertension among Secondary School Students in Ghana}

John Amoah
Ph.D. Candidate, Department of Community Health, Faculty of Medicine and Health Sciences,
University Putra Malaysia, Malaysia
Dr. Salmiah Md Said
Medical Lecturer, Department of Community Health, Faculty of Medicine and Health Sciences,
University Putra Malaysia, Malaysia
Lekhraj Rampal
Medical Lecturer, Department of Community Health, Faculty of Medicine and Health Sciences,
University Putra Malaysia, Malaysia
Rosliza Abdul Manaf
Medical Lecturer, Department of Community Health, Faculty of Medicine and Health Sciences,
University Putra Malaysia, Malaysia
Normala Ibrahim
Medical Lecturer, Department of Psychiatry, Faculty of Medicine and Health Sciences,
University Putra Malaysia, Malaysia

\begin{abstract}
:
Introduction: Worldwide prevalence of hypertension in 2014 was 22\% and is expected to increase.Prevalence of hypertension has been on the increase among adolescentsworldwide that persist during adulthood and has become a major public health problem. However, few studies on hypertension has been studied among secondary school students in Ghana. Materials and Methods: A cross sectional study design was conducted among 14-19-year-old secondary school students in two districts of Ghana. A validated self-administered questionnaire was used to collect data on socio-demographic characteristics, blood pressure, physical activity, weight and height. Two blood pressure measurements were taken for each student with Omron HBP-1100 automated BP (blood pressure) monitor while weight and height were taken with Tanita Model HD 309 and Seca body Meter Model 208 respectively. Data was analyzed with SPSS version 22 with descriptive statistics such as means, frequencies and percentages. Chi-Square test and Logistic regression were also utilized. Results: Prevalence of hypertension and elevated was $24.4 \%$. There were $11.0 \%$ students who were overweight while $2.2 \%$ were obese. Approximately $34.6 \%$ of the students were physically inactive. The independent variables in the logistics regression for hypertension was body mass index and physical activity. Conclusion:Risk factors of hypertension are increasing among students. A behavioral modification intervention should be implemented in secondary schools to reduce risk factors of hypertension among the students.
\end{abstract}

Keywords: Hypertension, elevated, BMI, physical activity, prevalence, secondary schoolstudents

\section{Introduction}

Hypertension is a major risk factor of cardiovascular disease (CVD) (Chiolero et al., 2013; Kuciene \& Dulskiene, 2014) and one of the most important contributors of morbidity and mortality in the world (Fortuna et al., 2015). The worldwide prevalence of hypertension in 2014 was around 22\% and it expected to grow to more than 500 million by 2025 (Boateng et al., 2015). Africa recorded the highest prevalence of hypertension (30\%) across the WHO regions while the lowest prevalence was in the Americas (18\%). There were 9.4 million deaths globally due to hypertension in 2010 (WHO, 2014). People with hypertension develop CVDs earlier than people with normal blood pressure (Rapsomaniki et al., 2014). Hypertension has been on the increase in Ghana. The number of reported new cases of hypertension between 1988 and 2007 rose by more than $1000 \%$ (Ofori-Asenso \& Garcia, 2015). Prevalence of adult hypertension in Ghana is at $48 \%$ and has ranked continuously among the top ten causes of outpatient morbidity, admission and death (Ghana Health Service, 2017). There were 964,724 newly reported cases of adult hypertension in outpatient departments across hospitals in Ghana in 2014 alone (Ghana Health Service, 2015).

Studies have shown that prevalence of hypertension has been on the increase among children which persist into adulthood(Chen \& Wang, 2008; Spagnolo et al., 2013). For instance, a study in America by Thompson et al. (2013) among children and adolescents indicated that between 1\% and 5\% were hypertensive. Other studies in United Arab Emirates (Abdulle et al., 2014) and Turkey (Demirci et al., 2013) have reported 16.6\% and 8.5\% hypertension respectively among 
children. A study in Ghana reported prevalence of 32.3\% and 4\% elevated and hypertensive respectively among the youth(Afrifa-Anane et al., 2015).The study further reported that physical activity among respondents was low and was associated with hypertension and BMI. However, very few studies on hypertension has been conducted among secondary school students in Ghana. Studies in this area are crucial to enable policy makers, researchers and the Ghanaian Ministry of Health to develop appropriate interventions to curb this situation. This study therefore aims to conduct a cross sectional survey on prevalence of elevated and hypertension among secondary school students in Ghana.

\section{Materials and Methods}

\subsection{Study Design}

This study was a cross sectional design carried out in Ghana from January to March, 2018.University Putra Malaysia (UPM/ TNCP/ 1.4.18.2 (JKEUPM) and the Kintampo Health Research Centre (KHRCIEC/ 2017-16) gave ethical clearance for this study. Permissions were obtained from the Ministry of Education, headmasters and parent teacher association chairmen of selected schools. Consents forms were given to all participating students and their parents/guardians before the study began. Code numbers were given to students and they were asked not to write their names or signatures on the questionnaires to ensure confidentiality.

\subsection{Sampling}

Students were recruited from four public secondary schools in two selected districts (Kintampo-North and Nkoranza-North) of the Brong Ahafo region by systematic random sampling. There were two public secondary schools in each of the two districts and all the schools were included in this study. Students were randomly selected from the lists of students from form one to three, given by headmasters, by random number generation by an independent biostatistician who was not involved in the study. The study would require a $90 \%$ power to defect an effect of 0.8 standard deviation at the $5 \%$ significance level taking into consideration a 10\% non-response rate.The sample size was 848 . Weight and height measurements were taken by trained health staff and measuring instruments were standardized on daily basis.

\subsection{Study Variables}

The analysed variables included age (years), gender, ethnicity, parent's education, elevated, hypertension, weight $(\mathrm{kg})$, height $(\mathrm{cm}), \mathrm{BMI}\left(\mathrm{kg} / \mathrm{m}^{2}\right)$, and physical activity.

\subsection{Dependent Variable}

\subsubsection{Hypertension}

Two blood pressure readings were taken with an Omron HBP-1100 automated BP monitor. The measurements were taken by trained health staff with the respondent comfortably seated for five minutes while legs were not crossed and the back and the arm supported before readings were taken. The respondent wasallowed to rest a minute after the first reading before the second measurement was taken(Pickering et al., 2005). The average of the two was used to classify

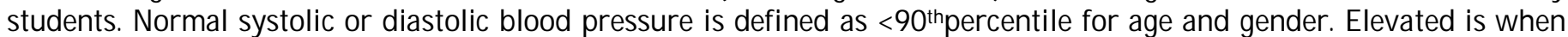
systolic or diastolic blood pressure is between $90^{\text {th }}$ and $95^{\text {th }}$ percentile. Hypertension is when systolic or diastolic blood pressure exceeds $95^{\text {th }}$ percentile(Fourth Report on Diagnosis , Evaluation , and Treatment of High Blood Pressure in Children and Adolescents, 2005).

\subsection{Independent Variables}

\subsubsection{Body Mass Index}

Weight and height measurements were the anthropometry measurements that were taken. Weight was taken with Tanita Model HD 309to the nearest $0.1 \mathrm{~kg}$. Students were asked to remove shoes and any item in the pocket. They were then asked to stand upright on the scale and the measurement was taken. Height was measured using a Seca Body Meter Model 208 to the nearest $0.1 \mathrm{~cm}$. Each respondent was asked to stand and looked straight, without shoe with heels resting together. Height measurement which appeared in the read-off area was recorded. Height and weight measurements were used to assess students $\mathrm{BMI}\left(\mathrm{BMI}=\right.$ Weight in $\mathrm{kg} /$ Height in $\left.\mathrm{m}^{2}\right)$ in terms of underweight, normal,

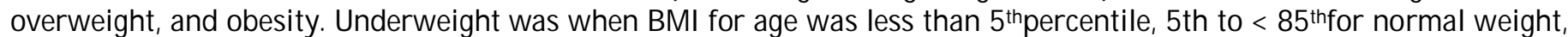
85th to <95th for overweight and $\geq 95^{\text {th }}$ represented obesity(Barlow, 2007; WHO, 2007).

\subsubsection{Physical Activity}

The physical activity questionnaire (PAQ-A) for secondary school adolescents(Kowalski et al., 2004) was used to measure physical activity levels. It was a seven-day recall instrument to assess general levels of physical activity among respondents. Each physical activity item was scored on a five-point scale. The value from one to five for each of the items used in the physical activity composite score, then the mean of the items which resulted in the final PAQ-A activity summary score.

\subsection{Statistical Analysis}

Descriptive data was analyzed using SPSS version 22. The level of significance for all the statistical tests was set at 0.05 . Frequencies and percentages were used to summarize data. For mean estimations, $95 \%$ confidence intervals (CI) was 
computed.Chi-Square test was used for categorical variables. Logistic regression was used to analyze contribution of independent variables on elevated and hypertension.

\section{Results}

3.1. Socio-Demographic Characteristics of Students

The study included 848 public secondary school students between the ages of 14 to 19 years old. Majority of the students (30.8\%) were between the ages of 17 years old while females made up $51.5 \%$ of the study sample. A total of $53.2 \%$ were of Akan ethnic background. Most students reported that their fathers (48.0\%) and mothers (51.5\%) had no formal education while $6.3 \%$ and $3.8 \%$ fathers and mothers respectively had tertiary education in Table 1.

\begin{tabular}{|c|c|c|}
\hline Socio-Demographic Factors & Frequency & $\mathbf{( \% )}$ \\
\hline Age (years) & & \\
\hline Mean (SD) & 16.99 & $(1.4)$ \\
\hline Range & & $(14-19)$ \\
\hline Gender & 411 & \\
\hline Male & 437 & $(48.5)$ \\
\hline female & & \\
\hline Ethnicity & 451 & $(53.2)$ \\
\hline Akan & 171 & $(20.9)$ \\
\hline Dagarte & 108 & $(12.7)$ \\
\hline Konkomba & 83 & $(9.8)$ \\
\hline Mo & 29 & $(3.4)$ \\
\hline Others & & \\
\hline Father's education & 407 & $(48.0)$ \\
\hline No formal education & 73 & $(8.6)$ \\
\hline Primary school & 170 & $(20.0)$ \\
\hline Junior high school & 145 & $(17.1)$ \\
\hline Secondary school & 53 & $(6.3)$ \\
\hline Tertiary & & \\
\hline Mother's education & 437 & $(51.5)$ \\
\hline No formal education & 115 & $(13.6)$ \\
\hline Primary school & 164 & $(19.3)$ \\
\hline Junior high school & 100 & $(11.8)$ \\
\hline Secondary school & 32 & $(3.8)$ \\
\hline Tertiary &
\end{tabular}

Table 1: Socio-Demographic Characteristics of Respondents

\subsection{Hypertension, BMI, and Physical Activity}

In Table 2, Prevalence of elevated and hypertension was $24.4 \%$. A total of $2.2 \%$ students were obese while $11.0 \%$ were overweight. On the other hand, $34.6 \%$ of the students were physically inactive with only $13.6 \%$ who were physically active (high).

\begin{tabular}{|c|c|c|}
\hline Variable & Frequency & (\%) \\
\hline Blood pressure & & \\
\hline Normal blood pressure & 641 & $(75.6)$ \\
\hline Elevated \& hypertension & 207 & $(24.4)$ \\
\hline BMI & & \\
\hline Underweight & 22 & $(2.6)$ \\
\hline Normal & 714 & $(84.2)$ \\
\hline Overweight & 93 & $(11.0)$ \\
\hline Obese & 19 & $(2.2)$ \\
\hline Physical activity & & \\
\hline Low & 293 & $(34.6)$ \\
\hline Medium & 440 & $(51.9)$ \\
\hline High & 115 & $(13.6)$ \\
\hline
\end{tabular}

Table 2: Blood Pressure, BMI, and Physical Activity of Respondents 


\subsection{Blood Pressure Distribution among BMI and Physical Activity}

In Table 3, $0.7 \%$ and 3.2\% obese and overweight students respectively were either elevated or hypertensive. On physical activity, $8.4 \%$ (low) and 12.5\% (medium) students were either elevated or hypertensive.

\begin{tabular}{|c|c|c|c|c|c|c|c|}
\hline Variable & \multicolumn{2}{|c|}{$\begin{array}{c}\text { Normal Blood } \\
\text { Pressure }\end{array}$} & \multicolumn{2}{c|}{$\begin{array}{c}\text { Elevated and } \\
\text { Hypertension }\end{array}$} & $\chi^{2}$ & $\mathrm{df}$ & $\mathrm{p}$ \\
\hline & Frequency & $(\%)$ & Frequency & $(\%)$ & & & \\
\hline BMI & & & & & 3.072 & 3 & 0.381 \\
\hline Underweight & 19 & $(2.2)$ & 3 & $(0.4)$ & & & \\
\hline Normal weight & 543 & $(64.0)$ & 171 & $(20.2)$ & & & \\
\hline Overweight & 66 & $(7.8)$ & 27 & $(3.2)$ & & & \\
\hline Obese & 13 & $(1.5)$ & 6 & $(0.7)$ & & & \\
\hline Physical activity & & & & & 0.205 & 2 & 0.903 \\
\hline Low & 222 & $(26.2)$ & 71 & $(8.4)$ & & & \\
\hline Medium & 334 & $(39.4)$ & 106 & $(12.5)$ & & & \\
\hline High & 85 & $(10.0)$ & 30 & $(3.5)$ & & & \\
\hline
\end{tabular}

Table 3: Blood Pressure versus BMI and Physical Activity

\subsection{Independent Contribution of Age, BMI, and Physical Activity on Hypertension among Students}

The results from logistic regression in Table 4 shows that BMI $(6.717, p=0.010)$ and physical activity $(5.013, p=$ 0.033 ) contributed significantly to elevated and hypertension, while age (1.956, $\mathrm{p}=0.162)$ was not.

\begin{tabular}{|c|c|c|c|c|c|c|c|}
\hline $\begin{array}{c}\text { Independent } \\
\text { Variables }\end{array}$ & $\begin{array}{c}\text { B } \\
\text { Coefficient }\end{array}$ & $\begin{array}{c}\text { Standard } \\
\text { Error }\end{array}$ & Wald & df & Exp (B) & 95\% CI (Exp B) & p \\
\hline Age & 0.081 & 0.058 & 1.956 & 1 & 1.085 & $(0.968-1.215)$ & 0.162 \\
\hline BMI & 0.077 & 0.030 & 6.717 & 1 & 1.080 & $(1.019-1.145)$ & 0.010 \\
\hline Physical activity & 0.056 & 0.071 & 5.013 & 1 & 1.137 & $(1.037-1.186)$ & 0.033 \\
\hline
\end{tabular}

Table 4: Independent Distribution of Age, Weight, and BMI on Blood Pressure in Students

\section{Discussion}

This study was conducted to find prevalence of elevated and hypertension among public secondary school students. Information on prevalence of hypertension among secondary school students seem to be scanty in Ghana. Hypertension and elevated among studentswere24.4\%. This was similar with studies among undergraduate students in Ghana (Gyamfi et al., 2018) but lower than a study in same country among the youth (Afrifa-Anane et al., 2015). Risk for CVD doubles with each increase of 20/10 $\mathrm{mmHg}$ (WHO, 2011)and therefore we recommend for screening of students on blood pressure and to monitor their BMI and physical activity. The other aspect of it is that many Ghanaians are unaware they have the condition. The Ministry of Health (Ministry of Health, 2012)has reported that up to about 70\% of Ghanaians who were detected to have hypertension were not aware of it. Out of this number, up to $13 \%$ only have their blood pressure controlled. Further, because the disease starts during childhood, measures should be put in place for prevention and or early detection.

The prevalence of obesity and overweight were $2.2 \%$ and $11 \%$ respectively. This was consistent with studies among secondary school students in Ghana(Kumah et al., 2015; Nyawornota et al., 2013). Obesity and overweight are major risk factor of hypertension, diabetes, and CVDs(Brady, 2016; Schutter et al., 2014). Results from logistic regression indicated that BMI contributes significantly to blood pressure. This suggest that children who are obese are at a higher risk of developing diseases and mortality in adulthood, poor mental, and physical health(Black et al., 2015; Robertson et al., 2016).There is evidence to suggest that lifestyle interventions on modifiable risk factors may prevent the onset of diabetes and future risk of obesity(Obirikorang et al., 2016).

This study showed that $34.6 \%$ of students were physically inactive. This is consistent with studies in Ghana by (Mogre et al., 2013). Physical inactivity is a major cause of overweight, obesity, and hypertension(WHO, 2010). The health benefits of physical activity includes prevention of obesity and hypertension(Hills et al., 2015). Physical activity declines during childhood and this declines faster during the adolescents'age(Dumith et al., 2011; Nader et al., 2008)and therefore is it critical to educate and motivate students to do some form of moderate to high physical activity on daily basis. Results also showed that low physical activity is a contributing factor of hypertension. A study by Afrifa-Anane et al. (2015) reported similar trends in his study among the youth. A meta-analysis of prospective studies has indicated doing some form of moderate to high physical activity reduces the risk of coronary heart disease and stroke by $20-30 \%$ and $10 \%-20 \%$ in men and women respectively(Li \& Siegrist, 2012).

\section{Conclusion}

It is clear from the findings of this study that risk factors of hypertension are increasing among students. Body mass index and physical activities were major contributors towards hypertension. It is highly recommended that students should be screened in the schools for early detection of hypertension and its risk factors. Finally, it is recommended for behavioral modification intervention in schools to reduce risk factors of hypertension among secondary school students. 


\section{References}

i. Abdulle, A., Al-junaibi, A., \&Nagelkerke, N. (2014). High Blood Pressure and Its Association with Body Weight among Children and Adolescents in the United Arab Emirates, 9(1), 1-5. doi:10.1371/journal.pone.0085129

ii. Afrifa-Anane, E., Agyemang, C., Codjoe, S. N. A., Ogedegbe, G., \& de-Graft Aikins, A. (2015). The association of physical activity, body mass index and the blood pressure levels among urban poor youth in Accra, Ghana. BMC Public Health, 15(1), 269. doi:10.1186/ s12889-015-1546-3

iii. Barlow, S. (2007). Expert Committee Recommendations Regarding the Prevention, Assessment, and Treatment of Child and Adolescent Overweight and Obesity: Summary Report. Pediatrics, 120(Suppl4), s164-s165. doi:10.1542/ peds.2007-2329C

iv. Black, J. A., Park, M., Gregson, J., Falconer, C. L., \& White, B. (2015). Child obesity cut-offs as derived from parental perceptions : British Journal of General Practice, 65(633), 234-239. doi:10.3399/ bjgp15X684385

v. Boateng, G. O., Luginaah, I. N., \&Taabazuing, M.-M. (2015). Examining the Risk Factors Associated With Hypertension Among the Elderly in Ghana. Journal of Aging and Health, 1-23. doi:10.1177/ 0898264315577588

vi. Brady, C. (2016). Decreasing Obesity and Obesity Stigma : Socio-Demographic Differences in Beliefs about Causes of and Responsibility for Obesity. Social Sciences, 5(12), 1-10. doi:10.3390/ socsci5010012

vii. Cawley, J., Meyerhoefer, C., Biener, A., Hammer, M., \&Wintfeld, N. (2015). Savings in Medical Expenditures Associated with Reductions in Body Mass Index Among US Adults with Obesity, by Diabetes Status. PharmacoEconomics, 33, 707-722. doi:10.1007/s40273-014-0230-2

viii. Chen, X., \& Wang, Y. (2008). Tracking of Blood Pressure From Childhood to Adulthood A Systematic Review and Meta - Regression Analysis. Epidemiology, 117, 3171-3180. doi:10.1161/ CIRCULATIONAHA.107.730366

ix. Chiolero, A., Bovet, P., \&Paradis, G. (2013). Screening for Elevated Blood Pressure in Children and Adolescents. JAMA Pediatr, 167(3), 266-273. doi:10.1001/jamapediatrics.2013.438

X. Demirci, H., Nuhoglu, C., Ursavas, I. S., Isildak, S., Basaran, E. O., \&Kılıc, M. Y. (2013). Obesity and asymptomatic hypertension among children aged 6 - 13 years living in Bursa, Turkey. Family Practice, 30, 629-633. doi:10.1093/ fampra/ cmt048

xi. Dumith, S. C., Gigante, D. P., Domingues, M. R., \& Kohl, H. W. (2011). Physical activity change during adolescence: A systematic review and a pooled analysis. International Journal of Epidemiology, 40(3), 685-698. doi:10.1093/ije/ dyq272

xii. Fortuna, R. J., Nagel, A. K., Rose, E., Mccann, R., Teeters, J. C., Quigley, D. D., Rocco, T. A. (2015). Effectiveness of a multidisciplinary intervention to improve hypertension control in an urban underserved practice. Journal of the American Society of Hypertension, 9(12), 966-974. doi:10.1016/j.jash.2015.10.004

xiii. Fourth Report on Diagnosis,Evaluation, and Treatment of High Blood Pressure in Children and Adolescents. (2005). http:/ / www.nhlbi.nih.gov

xiv. Ghana Health Service. (2015). 2014 Annual Report. Accra. http://www.ghanahealthservice.org/ghscategory.php.cid $=5$

xv. Ghana Health

Service.

(2017).

2016

Annual

Report.

Accra. http:/ / ghanahealthservice.org/ downloads/ GHS ANNUAL REPORT 2016 n.pdf

xvi. Gyamfi, D., Obirikorang, C., Acheampong, E., et., al. (2018). Prevalence of pre-hypertension and hypertension and its related risk factors among undergraduate students in a Tertiary institution, Ghana. Alexandria Journal of Medicine, 54(4), 475-480. doi:10.1016/ j.ajme.2018.02.002

xvii. Hills, A. P., Dengel, D. R., \&Lubans, D. R. (2015). Supporting Public Health Priorities: Recommendations for Physical Education and Physical Activity Promotion in Schools. Progress in Cardiovascular Diseases, 57(4), 368-374. doi:10.1016/j.pcad.2014.09.010

xviii. Kowalski, K. C., Crocker, P. R. E., \&Donen, R. M. (2004). The Physical Activity Questionnaire for Older Children (PAQ-C) and Adolescents (PAQ-A) Manual. College of Kinesiology, University of Saskatchewan. https:/ / www.researchgate.net/ publication/ 228441462

xix. Kuciene, R., \&Dulskiene, V. (2014). Associations of short sleep duration with prehypertension and hypertension among Lithuanian children and adolescents: a cross-sectional study. BMC Public Health, 14(255), 1-8. doi:10.1186/ 1471-2458-14-255

xx. Kumah, D. B., Akuffo, K. O., Abaka-Cann, J. E., Affram, D. E., \&Osae, E. A. (2015). Prevalence of Overweight and Obesity among Students in the Kumasi Metropolis. Journal of Nutrition and Metabolism, 2015, 1-4. doi:10.1155/2015/613207

xxi. Li, J., \&Siegrist, J. (2012). Physical activity and risk of cardiovascular disease-a meta-analysis of prospective cohort studies. International Journal of Environmental Research and Public Health, 9(2), 391-407. doi:10.3390/ijerph9020391

xxii. Ministry of Health. (2012). Strategy for the Management, Prevention and Control of Chronic Non- Communicable Diseases in Ghana 2012-2016. Accra. https:/ / www.mindbank.info/ item/ 1933

xxiii. Mogre, V., Aneyire, E. S., \&Gyamfi, E. K. (2013). Physical activity and BMI status of school-age children in Tamale, Northern Ghana. Pakistan Journal of Nutrition, 12(5), 484-490.

xxiv. Nader, P. R., Bradley, R. H., Houts, R. M., Mcritchie, S. L., \& O’Brien, M. (2008). Moderate-to-Vigorous Physical from Ages 9 to 15 Years. American Journal Association, 300(3), 295-305. doi:10.1001/ jama.300.3.295

xxv. Nyawornota, V. K., Aryeetey, R., Bosomprah, S., \&Aikins, M. (2013). An exploratory study of physical activity and over-weight in two senior high schools in the Accra Metropolis. Ghana Medical Journal, 47(4), 197-203. 
xxvi. Obirikorang, Y., Obirikorang, C.Anto, E. O., et., al. (2016). Knowledge and Lifestyle-Associated Prevalence of Obesity among Newly Diagnosed Type II Diabetes Mellitus Patients Attending Diabetic Clinic at KomfoAnokye Teaching Hospital , Kumasi, Ghana: A Hospital-Based Cross-Sectional Study. Diabetes Research, 2016, 1-10. doi:10.1155/2016/9759241

xxvii. Ofori-asenso, R., \& Garcia, D. (2015). Cardiovascular diseases in Ghana within the context of globalization, 1-11. doi:10.3978/j.issn.2223-3652.2015.09.02

xxviii. Pickering, T. G., Hall, J. E., Appel, L. J., et., al. (2005). Recommendations for blood presure measurement in humans and experimental animals. Part 1: Blood pressure measurement in humans. Hypertension, 45, 142-161. doi:10.1161/ 01.HYP.0000150859.47929.8e

xxix. Rapsomaniki, E., Timmis, A., George, J., et., al. (2014). Blood pressure and incidence of twelve cardiovascular diseases : lifetime risks, healthy life-years lost, and age-specific associations in 1.25 million people. Lancet, 383, 1899-1911. doi:10.1016/ S0140-6736(14)60685-1

xxx. Robertson, W., Murphy, M., \& Johnson, R. (2016). Evidence base for the prevention and management of child obesity. Paediatrics and Child Health, 1-7. doi:10.1016/ j.paed.2015.12.009

xxxi. Schutter, A. De, Lavie, C. J., \&Milani, R. V. (2014). The Impact of Obesity on Risk Factors and Prevalence and Prognosis of Coronary Heart Disease - The Obesity Paradox. Progress in Cardiovascular Diseases, 56(4), 401408. doi:10.1016/j.pcad.2013.08.003

xxxii. Spagnolo, A., Giussani, M., Ambruzzi, A. M.et., al. (2013). Focus on prevention, diagnosis and treatment of hypertension in children and adolescents. Italian Journal of Pediatrics, 39(20), 1-18. doi:10.1186/ 1824-7288-3920

xxxiii. Thompson, M., Dana, T., Bougatsos, C., Blazina, I., \& Norris, S. L. (2013). Screening for Hypertension in Children and Adolescents to Prevent Cardiovascular Disease abstract. American Academy of Padiatrics, 131(3), 490-525. doi:10.1542/ peds.2012-3523

xxxiv. World Health Organization. (2007). BM-for-Age (15-19 years). Retrieved November 20, 2016, http:/ / www.who.int/ growthref/ who2007_bmi for age/ en/

xxxv. World Health Organization. (2010). Global recommendations on physical activity for health. http:/ / apps.who.int/ iris/ bitstream/ 10665/ 44399/ 1/ 9789241599979 eng.pdf

xxxvi. World Health Organization. (2011). A prioritized research agenda for prevention and control of noncommunicable diseases, World Health Organization, Geneva. http:/ / apps.who.int/ iris/ bitstream/ 10665/ 44569/ 1/ 9789241564205_eng.pdf

xxxvii. World Health Organization. (2014). Global status report on noncommunicable diseases 2014 "Attaining the nine global noncommunicable diseases targets; a shared responsibility." Geneva. http:/ / www.who.int 